\title{
Duality of Anti-nutritional Factors in Pulses
}

\section{Anjana Agarwal ${ }^{\star}$}

Nutritionist and Aromatherapist, SNDT Women's University, Mumbai, India

*Corresponding author: Anjana Agarwal, Nutritionist and Aromatherapist, SNDT Women's University, Mumbai, India, E-mail: dranjanaagarwal@gmail.com

Rec date: 28 March, 2016; Acc date: 29 March, 2016; Pub date: 31 March, 2016

Copyright: () 2016 Agarwal A. This is an open-access article distributed under the terms of the Creative Commons Attribution License, which permits unrestricted use, distribution, and reproduction in any medium, provided the original author and source are credited.

\section{Editorial}

Plants naturally develop inherent defense mechanism by synthesizing of secondary metabolites to protect themselves from pests and pathogens in adverse environmental conditions. Some metabolites or substances pose deleterious effects on animals and human beings, thus referred as 'Anti-Nutritional Factors (ANFs) and the same have also shown beneficial impact on human health. Hence judicious processing of ANF may prove an asset in agriculture, pharmacology and nutrition world.

Besides being rich in protein, carbohydrate, fiber, vitamins and minerals, pulses, a subgroup of legume family, are rich in antinutritional factors (ANF). On one hand, ANFs interfere with the absorption of many vital nutrients and limit their utilization and on the other hand they are reported to be hypocholesterolemic, anticancer and anti-atherosclerotic. Pulses are dried seeds and can be stored for several months without losing their nutritional value. They necessarily need cooking and are being used in almost all cuisines of the world.

The United Nations has declared 2016 as "International Year of Pulses" (IYP) to heighten public awareness of the nutritional benefits of pulses aiming to bring more food and nutrition security. Pulses are good choice for the malnourished population particularly in developing countries where most people subsist on plant food sources for their energy and nutrient requirement.

Primarily pulses are hard seeds due to which they cannot be consumed without processing. Traditional processing methods are dehulling, milling, soaking, cooking, pressure cooking, roasting, germination and fermentation; and in disguise, these methods reduce ANFs to varying degrees. However, modern technological methods like fractionation, ultrafiltration extraction and extrusion can modulate them as functional food or nutraceutical ingredients.

Common anti-nutritional factors (ANFs) are protease inhibitors (trypsin inhibitor), amylase inhibitors, lectins, saponins, phytic acid, and the oligosaccharides of raffinose family. They vary greatly from pulse to pulse; variety to variety and further influenced by season, processing method, storage and level of insect infestation.

Among protease inhibitors, trypsin inhibitor is of high concern because it significantly reduces the digestibility of proteins and responsible for reducing the sulphur containing amino acids like methionine and cysteine in pulses. It also acts an insecticide. Moth bean contains large amount of trypsin inhibitor; autoclaving can destroy it. Reports showed that germination of cowpea for 5 days can significantly reduce the activity of trypsin inhibitor (TI).

Raffinose family of oligosaccharides (RFO) consisting of raffinose, stachyose, verbascose sugars, are commonly found in pulses and is associated with flatulence (abdominal gas production) with pulse consumption. These sugars ferment in the large intestine and cause flatulence that is uncomfortable and unacceptable. RFOs also interfere with the digestion of other nutrients. Thermal treatment, use of certain spices and germination significantly reduced RFOs. On contrary, they reduce the risk of intestinal cancer; increase stool weight and frequency. During germination RFO serve as a carbon source for the new seedlings.

Lectins, the glycoproteins prevent absorption of digestive end products in small intestine by binding with different sugar moieties in intestinal wall. When lima beans containing large quantity of lectins (hemaglutinnins) are consumed raw or in large amount can coagulate red blood cells (RBCs). Lectins are responsible for specific blood group in humans; function in mitotic division and demolish cancer cells. Some lectins are useful in genetic engineering; thereby lectin- free crop can be cultivated enhancing resistance to insects.

Soap like formation in pulses is due to saponin. High concentration of saponin in the diet can cause hemolysis of RBCs. They interact with biomembranes causing pores and holes in them leading to apoptosis (cell death). Clinical studies have suggested that saponins decrease blood lipids, lower cancer risks, and lower blood glucose response. A high saponin diet can be used in the inhibition of dental caries. However, canned navy beans do not show hemolytic effect.

Amylase inhibitors slow down the digestion of starch and alter the sugar response to insulin which can be exploited for diabetes.

Phytates are important source of phosphate and minerals which are required during germination. But they form complex with iron, zinc, magnesium and calcium in the digestive tract and cause mineral deficiency in human and animals. They are responsible for 'hard tocook' phenomena in pulses. They can be reduced by addition of enzymes, phytase and germination. Phytic acid level in legumes range from 0.4 to $6.4 \%$ by weight and they are antioxidant and anti-cancer agent.

Phenol compounds determine the colour of the flower of the plant and taste bitter. They reduce the bioavailability of the minerals (zinc). They form complex with protein molecules and decrease their solubility thus digestibility. However, phenol compounds are useful as anti-cancer and anti-atherosclerotic.

Vicine and convicine present in faba beans are responsible for acute hemolytic disease (favism). They also help in reducing cholesterol.

A neurotoxin called B-N-oxalyl diaminopropionic acid (BOAA) is found in Lathyrus sativus (kesari dal). On continuous consumption of it for more than six months in large amount can paralyse lower limbs in men as this substance interface with formation of normal collagen in connective tissues.

Anti-nutritional factors (ANF) in pulses are great challenge to the nutritionists and food technologists for its nutritional concerns and 
Citation: Agarwal A (2016) Duality of Anti-nutritional Factors in Pulses. J Nutr Disorders Ther 6: e124. doi:10.4172/2161-0509.1000e124

Page 2 of 2

utility. Conserving the nutritional richness, pulses could have been a malnutrition and by virtue of several bioactive compounds pulses are very good commodity to tackle the global problem of hunger and functional ingredient in health and disease. 